IJBPAS, November, 2020, 9(11): 3075-3087

ISSN: 2277-4998

International Journal of Biology, Pharmacy

and Allied Seiences (IJBPAS)

'A Bridge Betusen Caboratory and Qender'

Www.iibpas.com

\title{
EFFECT OF DIETARY PROTEIN LEVELS ON GROWTH AND MORPHOMETRICS OF STRIPED CATFISH PANGASIANODON HYPOPHTHALMUS (SAUVAGE, 1878) FROM PAKISTAN
}

\begin{abstract}
MALIK T AND NAEEM M*
Institute of Pure and Applied Biology, Bahauddin Zakariya University, Multan, Pakistan *Corresponding Author: Naeem M: E Mail: muhammadnaeem@bzu.edu.pk; +923337378881

Received 15 ${ }^{\text {th }}$ Dec. 2019; Revised 14 ${ }^{\text {th }}$ Feb. 2020; Accepted 20 ${ }^{\text {th }}$ May 2020; Available online $1^{\text {st }}$ Nov. 2020 https://doi.org/10.31032/IJBPAS/2020/9.11.5274

ABSTRACT
\end{abstract}

In recent study a 90 days feeding trial was conducted to evaluate the effect of dietary protein ratios on growth and morphometry in Pangasianodon hypophthalmus. Three commercially available diets with crude protein ratios 20\% (T1), 25\% (T2) and 30\% (T3), by Supreme Aqua Feeds, were fed to $P$. hypophthalmus fry by initial weight and length $0.30 \mathrm{~g}$ and $2.90 \mathrm{~cm}$ respectively. Average final weight $(26.84 \mathrm{~g})$, average final length $(14.51 \mathrm{~cm})$, survival $(98 \%)$ and Ccondition factor (0.88) were high for 30\% CP (crude protein) Diet. FCR (feed conversion ratio) improved with increase in dietary protein moving from $\mathrm{T} 1$ to $\mathrm{T} 3$. Various morphometric parameters i.e. total length (TL), fork length (FL) standard length (SL), head length (HL), body depth (BD), body girth (BG), dorsal fin base (DFB), dorsal fin length (DFL), $2^{\text {nd }}$ dorsal fin base $\left(2^{\text {nd }} D F B\right), 2^{\text {nd }}$ dorsal fin length $\left(2^{\text {nd }} D F L\right)$, pelvic fin length $(P v F L)$, pectoral fin length $(P t F L)$, anal fin length (AFL), anal fin base(AFB), caudal fin length(CFL), head width (HW), eye diameter (ED), Inter-orbital distance (Int.OD), pre-orbital length (POL) of P. hypophthalmus were studied to know their correlation to size (TL). The favorable growth environment supported the survival of fish. However all diets improved the growth of fish, the $30 \% \mathrm{CP}$ which resulted in best growth performance was probably adequate to meet the dietary protein needs of $P$. hypophthalmus fry in recent study.

Keywords: Pangasianodon hypophthalmus, crude protein ratios, commercial feed, growth performance, morphometrics 


\section{INTRODUCTION}

Pangasianodon hypophthalmus is a benthopelagic omnivorous species that lives at a $\mathrm{pH}$ of $6.5-7.5$ and a temperature range of $22-26^{\circ} \mathrm{C}$ [1]. Fish is one of the best source of cheap meat and protein necessity to half of the world's population that influences the ability of farmed fish to show their genetic prospective for reproduction and growth [2]. Currently, maximum farms use commercially produced feed [3] and the use of consumed protein in fish feed enhances the growth presentation [4]. Protein in fish farming is the chief costly component of artificial feed, it makes sense to only include that much, which is essential for usual preservation mandate and growth, extra protein is waste both biologically and economically [5] thus, cost-effective and well-adjusted diet had to be utilized for improving the fish production [6] and growth of fish [7]. The growth rate and feed conversion ratio (FCR) had an obvious impact by volume and value of diet fed by fish [6]. To identify and classify fishes, taxonomists and ichthyologists use various morphometric characteristics, including body length, head length, depth and fin lengths, standard and fork length of the fish [8]. It offers fundamental information, being useful to compare between-region comparisons growth, to estimate the length of an individual fish of known weight or vice versa [9], to evaluate the isometric and allometric growth pattern in fish [10]. Several factors, like age, food availability and its source, physiochemical parameters of water for fish growth and sexual maturity of fish, may influence on K-value [11]. Furthermore, it can also be used in assessing the well-being of fish [12]. Hence, the purpose of recent study was to evaluate the impact of commercial protein diet of three levels $(20 \%$, $25 \%$ and $30 \%$ crude protein) at $P$. hypophthalmus on account of growth and morphometrics.

\section{MATERIALS AND METHODS}

\section{Experimental site}

The feeding trial was demonstrated at the Fis h Farm Facility Tawakkal Nagar, Muzaffar G arh in

Cemented ponds (indoor) for 90 days. The fish selected for experiment was Pangasianodon hypophthalmus fry. The average initial wet body weight and the average initial body length were measured at the time of stocking. The area for each cemented nursery pound was $2.48 \mathrm{~m}^{2}$.

\section{Experimental design}

Three treatments were designed on the grounds of three percentages of 
commercially available protein diet by Supreme Aqua Feeds. As T1 $=20 \%$, T2 $=25 \%$ and $\mathrm{T} 3=30 \%$ crude protein diet. The ponds were washed with an antiseptic, then each treatment was stocked with 100 fish fry (50 each replicate). The water quality was monitored and changed periodically to avoid turbidity, aerators were used to keep oxygen supply constant. The daily feed ratio was $5 \%$ of body biomass.

\section{Determination of Growth profile}

At the end of feeding trial all fish were harvested and brought to the fisheries lab at Institute of Pure and Applied Biology (Zoology Division) Bahauddin Zakariya University, Multan. The final weight and length and various growth parameters were measured for observing growth efficiency of $P$. hypophthalmus raised at commercial protein diet.

\section{Determination of Morphometrics}

In recent study 60 randomly selected fish specimens were used, 20 from each treatment. Morphometry was demonstrated at completion of growth experiment. At arrival in the laboratory, the fish were weighted (g) on an electric digital balance and length measurements $(\mathrm{cm})$ were taken. The morphometric parameters FL, SL, HL, BD, BG，DFB，DFL，2 $2^{\text {nd }} D F B ， 2^{\text {nd }} D F L ， P v F L$, PtFL, AFL, AFB, CFL, HW, ED, Int.OD, and POL of $P$. hypophthalmus were measured.

\section{Data analysis}

Using descriptive statistics growth data was expressed as Mean values. Whereas, correlation of morphometric parameters towards body size (TL) was calculated by linear regression, the data was transformed into logarithm by using MS-Excel 2016. This logarithm transformed data was then used for further analysis of total length relationships by; [ $\log Y=a+b \log X]$. Where ' $a$ ' and ' $b$ ' are constants of the relationship, representing the intersect part of the "Y" axis and slope of the equation, respectively.

\section{RESULTS}

\section{Growth performance of $\boldsymbol{P}$. hypophthalmus}

The Growth parameters of Pangasianodon hypophthalmus in various treatments i.e. T1 $(20 \%), \mathrm{T} 2(25 \%)$ and T3 (30\%) are shown in Table 1. The commercial crude protein diet by Supreme Aqua Feeds significantly affected the experimental groups. There was no significant difference between initial weight and length of all treatments but final weight and length increases for all groups under concern. Whereas the significant difference was observed among the final weight and length of all treatments. The final length and weight i.e. $26.84 \mathrm{~g}$ and $14.51 \mathrm{~cm}$ were observed to be highest in T3. Body 
weight (W) and Total length (TL) rises in order $\mathrm{T} 3>\mathrm{T} 2>\mathrm{T} 1$. The live weight gain i.e. $2600.32 \mathrm{~g}$ was highest in T3 in contrast to T2 (2259.06g) and T1 (2048.3g). The condition factor was observed as T1 $0.87, \mathrm{~T} 20.83$ and T3 0.88 however T3 was found significantly high. The values for FCR were identified as 1.29, 1.26 and 1.23 for $\mathrm{T} 1, \mathrm{~T} 2$ and $\mathrm{T} 3$ respectively. However significantly lower value was observed in $\mathrm{T} 3$ as compare to $\mathrm{T} 1$ and T2. Highest production and the survival percentage was 11.650 gram $/ \mathrm{m}^{2} / 90$ days and 98\% for T3 (30\% CP) respectively (Table 1 ).

\section{External Morphometric Variants of P.hypophthalmus}

The Table 2 signifies the mean ( \pm Standard Deviation) and range values for morphometric variants in three treatments of Pangasianodon hypophthalmus. The highest values for mean Body weight (wet) (WW) 26.84 \pm 2.38 , Condition factor $(\mathrm{K}) 0.88 \pm 0.05$, Total length (TL) $14.51 \pm 0.54$, Fork length (FL) $13.08 \pm 0.81$, Standard length (SL) $12.42 \pm 0.65$, Body depth (BD) $4.54 \pm 0.25$ and Body girth (BG) $9.09 \pm 0.50$ were all observed in group fed at 30\% CP diet (T3). Moreover, Dorsal fin length (DFL) $2.22 \pm 0.18$, Pelvic fin length (PvFL) 1.52 \pm 0.10 , Anal fin length (AFL) 1.86 \pm 0.18 , Anal fin base (AFB) $4.04 \pm 0.18$ and Caudal fin length (CFL) $2.54 \pm 0.30$ were also found to be highest in T3. In Table $\mathbf{3}$ a highly significant and positive correlation was recorded for wet body-weight (w.W) and most of parameters towards TL. The regression analysis among $\log$ of various morphometric parameters of $P$. hypophthalmus toward log total length $(\mathrm{cm})$ for three diet treatments is shown in Table 4. The Correlation coefficient values for all the three treatments ranged from $0.84-0.90$ were highly significant $(\mathrm{P}<0.001)$ between $\mathrm{W}$ and TL in $\log$ transformed data. The regression coefficient i.e. b value is ranged from $2.00-2.97$ that is near to an ideal slope value $(b=3)$. Therefore growth was seen to be isometric in T1 and negative allometry in T2 and T3. P. hypophthalmus. The $\log$ Condition factor specifies a significant relation (0.644) towards T3. Were as other morphometric parameters express highly significant relations towards body size (log TL), expressing good effect of feed at morphometry (Table 4). 
Table 1: Growth demonstration of Pangasianodon hypophthalmus (Sauvage, 1878) reared 90 days at various protein ratios of commercial diet in cemented fish-ponds

\begin{tabular}{|c|c|c|c|}
\hline Parameters & $\begin{array}{c}\text { T-1 } \\
20 \% \text { CP }\end{array}$ & $\begin{array}{c}\text { T-2 } \\
25 \% \text { CP }\end{array}$ & $\begin{array}{c}\text { T-3 } \\
30 \% \text { CP }\end{array}$ \\
\hline Rearing-period & 90(days) & 90(days) & 90(days) \\
\hline No. of-Stock & 100 & 100 & 100 \\
\hline FeedingRate/BodyWeight & $5 \%$ & $5 \%$ & $5 \%$ \\
\hline Initial-Weight(g)(Average) & 0.30 & 0.30 & 0.30 \\
\hline Final Weight(g)(Average) & 24.38 & 25.16 & 26.84 \\
\hline Live weight gain (g) & 2048.3 & 2259.06 & 2600.32 \\
\hline Average Initial length (cm) & 2.90 & 2.90 & 2.90 \\
\hline Average Final length $(\mathrm{cm})$ & 14.06 & 14.47 & 14.51 \\
\hline Length gain $(\mathrm{cm})$ & 11.16 & 11.57 & 11.61 \\
\hline Daily Weight Gain (g) & 22.76 & 25.10 & 27.70 \\
\hline Survival (\%) & $85 \%$ & $91 \%$ & $98 \%$ \\
\hline Average Initial-Condition- Factor & 1.46 & 1.46 & 1.46 \\
\hline Average Final-Condition- Factor & 0.87 & 0.83 & 0.88 \\
\hline FCR(FeedConversionRatio) & 1.29 & 1.26 & 1.23 \\
\hline Protein efficiency ratio & 3.86 & 3.18 & 2.71 \\
\hline Specific Growth rate & 2.230 & 2.129 & 2.169 \\
\hline Production $\mathrm{g} / \mathrm{m}^{2} / 90 d a y s$ & 9.177 & 10.121 & 11.650 \\
\hline
\end{tabular}

Table 2: Average-Values Analysis for External-Morphology of P.hypophthalmus $(\mathrm{n}=20)$

\begin{tabular}{|c|c|c|c|c|c|c|}
\hline \multirow[t]{2}{*}{ Body Measurements } & \multicolumn{2}{|c|}{ T1(20\%) } & \multicolumn{2}{|c|}{ T2(25\%) } & \multicolumn{2}{|c|}{ T3(30\%) } \\
\hline & Average \pm S.D & Ranges & Average \pm S.D & Ranges & Average \pm S.D & Ranges \\
\hline BodyWeight(Wet)(WW)g & $24.38 \pm 3.13$ & $19.10-29.00$ & $25.16 \pm 3.87$ & $21.70-33.20$ & $26.84 \pm 2.38$ & $23.90-31.30$ \\
\hline Condition Factor(K) & $0.87 \pm 0.06$ & 0.75-1.00 & $0.83 \pm 0.05$ & $0.76-0.99$ & $0.88 \pm 0.05$ & 0.81-0.97 \\
\hline Total length(TL)cm & $14.06 \pm 0.52$ & 13.10-14.70 & $14.47 \pm 0.69$ & 13.70-15.90 & $14.51 \pm 0.54$ & $13.50-15.20$ \\
\hline Fork-Length(FL)cm & $12.56 \pm 0.53$ & $11.60-13.20$ & $12.52 \pm 0.28$ & $12.20-13.10$ & $13.08 \pm 0.81$ & $11.50-14.00$ \\
\hline Standard Length(SL)cm & $11.90 \pm 0.38$ & $11.20-12.40$ & $11.72 \pm 0.27$ & $11.40-12.20$ & $12.42 \pm 0.65$ & $11.00-13.30$ \\
\hline Head-Length(HL)cm & $2.74 \pm 0.12$ & $2.60-2.90$ & $2.92 \pm 0.23$ & $2.50-3.20$ & $2.66 \pm 0.26$ & $2.10-2.90$ \\
\hline Body-Depth(BD)cm & $3.68 \pm 0.22$ & 3.25-3.95 & $3.75 \pm 0.12$ & $3.55-3.90$ & $4.54 \pm 0.25$ & 4.25-4.95 \\
\hline Body-Girth(BG)cm & $7.36 \pm 0.44$ & $6.50-7.90$ & $7.50 \pm 0.24$ & $7.10-7.80$ & $9.09 \pm 0.50$ & $8.50-9.90$ \\
\hline DorsalFinLength(DFL) cm & $2.20 \pm 0.17$ & $1.90-2.40$ & $2.12 \pm 0.18$ & $1.80-2.30$ & $2.22 \pm 0.18$ & $1.90-2.40$ \\
\hline Dorsal-FinBase(DFB) cm & $0.98 \pm 0.13$ & 0.80-1.10 & $0.90 \pm 0.07$ & 0.80-1.00 & $0.94 \pm 0.08$ & 0.90-1.10 \\
\hline $\begin{array}{l}2^{\text {nd }} \text { DorsalFinLength } \\
\left(2^{\text {nd }} D F L\right) c m\end{array}$ & $0.60 \pm 0.06$ & $0.50-0.70$ & $0.58 \pm 0.04$ & $0.50-0.60$ & $0.57 \pm 0.05$ & $0.50-0.60$ \\
\hline $2^{\text {nd }}$ DorsalFinBase $\left(2^{\text {nd }}\right.$ DFB $) \mathrm{cm}$ & $0.23 \pm 0.05$ & $0.20-0.30$ & $0.28 \pm 0.04$ & $0.20-0.30$ & $0.28 \pm 0.04$ & $0.20-0.30$ \\
\hline Pectoral-Fin Length (PtFL)cm & $1.88 \pm 0.10$ & $1.70-2.00$ & $1.94 \pm 0.11$ & $1.80-2.10$ & $1.92 \pm 0.21$ & $1.50-2.20$ \\
\hline Pelvic-FinLength (PvFL)cm & $1.48 \pm 0.07$ & $1.40-1.60$ & $1.48 \pm 0.08$ & $1.40-1.60$ & $1.52 \pm 0.10$ & $1.40-1.70$ \\
\hline AnalFinLength(AFL) cm & $1.70 \pm 0.14$ & $1.60-1.90$ & $1.50 \pm 0.13$ & $1.30-1.70$ & $1.86 \pm 0.18$ & $1.50-2.10$ \\
\hline Anal-Fin Base (AFB) cm & $3.86 \pm 0.21$ & $3.50-4.10$ & $3.78 \pm 0.16$ & $3.50-4.00$ & $4.04 \pm 0.18$ & $3.70-4.20$ \\
\hline Caudal-Fin Length (CFL)cm & $2.36 \pm 0.20$ & $2.00-2.60$ & $2.38 \pm 0.09$ & $2.30-2.50$ & $2.54 \pm 0.30$ & $2.10-2.90$ \\
\hline Head-Width(HW)cm & $1.90 \pm 0.22$ & $1.70-2.30$ & $2.70 \pm 0.23$ & $2.30-2.90$ & $2.60 \pm 0.26$ & $1.90-2.80$ \\
\hline EyeDiameter(ED)cm & $0.58 \pm 0.04$ & $0.50-0.60$ & $0.57 \pm 0.05$ & $0.50-0.60$ & $0.58 \pm 0.04$ & $0.50-0.60$ \\
\hline $\begin{array}{c}\text { InterOrbital } \\
\text { Distance(Int.OD)cm }\end{array}$ & $3.07 \pm 0.05$ & 3.00-3.10 & $3.54 \pm 0.08$ & $3.40-3.60$ & $3.08 \pm 0.04$ & 3.00-3.10 \\
\hline Pre-Orbital Length (POL)cm & $\mathbf{0 . 5 3} \pm \mathbf{0 . 0 7}$ & $0.40-0.60$ & $0.87 \pm 0.05$ & $\begin{array}{l}0.80-0.90 \\
\end{array}$ & $0.48 \pm 0.04$ & $0.40-0.50$ \\
\hline
\end{tabular}


Table 3: Analysing Regression among different morphometric considerations of Pangasianodon hypophthalmus toward total length $(\mathrm{cm})$ for $\mathrm{T} 1=\mathbf{2 0} \%$. $(\mathrm{n}=\mathbf{2 0})$

\begin{tabular}{|c|c|c|c|c|c|c|c|}
\hline Equations. & $\begin{array}{c}\text { Diet } \\
\text { Variables. }\end{array}$ & $\begin{array}{c}\text { [Relations } \\
\mathbf{a} \\
\end{array}$ & $\begin{array}{c}\text { arameters. } \\
\text { b }\end{array}$ & $\begin{array}{c}\mathrm{a} \\
(\mathrm{CI} .95 \%) \\
\end{array}$ & $\begin{array}{c}\text { b } \\
(\mathrm{CI} .95 \%) \\
\end{array}$ & r. & $\mathbf{r}^{2}$ \\
\hline \multirow[t]{3}{*}{$\mathbf{W}=(\mathbf{a}+\mathbf{b}) \mathbf{T L}$} & T1(20\%) & -47.1873 & 5.09 & $69.366--25.009$ & $3.514-6.667$ & $0.848 * * *$ & 0.719 \\
\hline & T2(25\%) & -48.7850 & 5.11 & $-65.741--31.829$ & $3.941-6.283$ & $0.908 * * *$ & 0.824 \\
\hline & T3(30\%) & -28.0857 & 3.79 & $-44.298--11.873$ & $2.669-4.903$ & $0.859 * * *$ & 0.738 \\
\hline \multirow[t]{3}{*}{$K=(a+b) T L$} & T1(20\%) & 0.9077 & -0.002 & $0.108-1.707$ & $-0.059-0.054$ & $0.021 \mathrm{~ns}$ & 0.0004 \\
\hline & T2(25\%) & 0.9883 & -0.01 & 0.438 - 1.539 & $-0.049-0.027$ & 0.144 ns & 0.021 \\
\hline & T3(30\%) & 1.7760 & -0.06 & $1.253-2.299$ & $-0.098--0.026$ & $0.648 * *$ & 0.420 \\
\hline \multirow[t]{3}{*}{$F . L=(a+b) T L$} & T1(20\%) & 2.3782 & 0.72 & $-2.656-7.413$ & 0.366 - 1.082 & $0.708 * * *$ & 0.501 \\
\hline & T2(25\%) & 7.6249 & 0.34 & $5.923-9.327$ & $0.221-0.456$ & $0.819 * * *$ & 0.670 \\
\hline & T3(30\%) & -0.0738 & 0.91 & $-8.618-8.470$ & $0.318-1.495$ & $0.606 * *$ & 0.368 \\
\hline \multirow[t]{3}{*}{$\mathrm{SL}=(\mathbf{a}+\mathbf{b}) \mathrm{TL}$} & T1(20\%) & 2.9750 & 0.63 & 0.411 - 5.539 & $0.452-0.817$ & $0.865 * * *$ & 0.748 \\
\hline & T2(25\%) & 6.7281 & 0.35 & $5.407-8.049$ & $0.254-0.436$ & $0.882 * * *$ & 0.778 \\
\hline & T3(30\%) & -2.9992 & 1.06 & $-7.172-1.173$ & $0.776-1.351$ & $0.878 * * *$ & 0.770 \\
\hline \multirow[t]{3}{*}{$\mathrm{HL}=(\mathbf{a}+\mathbf{b}) \mathrm{TL}$} & T1(20\%) & 0.8013 & 0.14 & $-0.458-2.060$ & $0.048-0.227$ & $0.606 * *$ & 0.367 \\
\hline & T2(25\%) & -0.4907 & 0.24 & $-2.240-1.259$ & $0.115-0.357$ & $0.695 * * *$ & 0.483 \\
\hline & T3(30\%) & -3.2002 & 0.40 & $-5.098--1.303$ & $0.273-0.535$ & $0.837 * * *$ & 0.701 \\
\hline \multirow[t]{3}{*}{$\mathbf{B D}=(\mathbf{a}+\mathbf{b}) \mathrm{TL}$} & T1(20\%) & -1.0802 & 0.34 & $-2.798-0.637$ & $0.216-0.460$ & $0.808 * * *$ & 0.653 \\
\hline & T2(25\%) & -0.3296 & 0.34 & $-1.285-0.626$ & $0.271-0.403$ & $0.930 * * *$ & 0.865 \\
\hline & T3(30\%) & 2.0811 & 0.11 & 0.716 - 3.446 & $0.021-0.209$ & $0.518^{*}$ & 0.268 \\
\hline \multirow[t]{3}{*}{$\mathbf{B G}=(a+b) T L$} & T1(20\%) & -2.1604 & 0.68 & $-5.596-1.275$ & $0.433-0.921$ & $0.808 * * *$ & 0.653 \\
\hline & T2(25\%) & -0.6592 & 0.67 & $-2.571-1.252$ & $0.542-0.806$ & $0.930 * * *$ & 0.865 \\
\hline & T3(30\%) & 4.1622 & 0.23 & $1.432-6.892$ & $0.042-0.418$ & $0.518^{*}$ & 0.268 \\
\hline \multirow[t]{3}{*}{$\mathbf{D F L}=(\mathbf{a}+\mathbf{b}) \mathrm{TL}$} & T1(20\%) & 0.4794 & 0.12 & $-1.610-2.569$ & $-0.026-0.271$ & $0.378 \mathrm{~ns}$ & 0.143 \\
\hline & T2(25\%) & -0.1452 & 0.16 & $-1.645-1.354$ & $0.053-0.260$ & $0.599 * *$ & 0.359 \\
\hline & T3(30\%) & -1.9194 & 0.29 & $-3.222--0.617$ & $0.196-0.375$ & $0.844 * * *$ & 0.713 \\
\hline \multirow[t]{3}{*}{$2^{\text {nd }} D F L=(a+b) T L$} & T1(20\%) & -0.4105 & 0.07 & $-1.120-0.299$ & $0.021-0.122$ & $0.577 * *$ & 0.332 \\
\hline & T2(25\%) & 0.0708 & 0.03 & $-0.320-0.461$ & $0.008-0.062$ & $0.539 *$ & 0.290 \\
\hline & T3(30\%) & 0.5296 & 0.002 & $-0.122-1.181$ & $-0.042-0.047$ & $0.027^{\text {ns }}$ & 0.0007 \\
\hline \multirow[t]{3}{*}{ Pt.FL $=(a+b) T L$} & T1(20\%) & -0.2280 & 0.15 & $-0.995-0.539$ & $0.095-0.204$ & $0.806 * * *$ & 0.649 \\
\hline & T2(25\%) & 0.9913 & 0.07 & $-0.051-2.034$ & $-0.006-0.138$ & $0.411^{\text {ns }}$ & 0.169 \\
\hline & T3(30\%) & 0.1089 & 0.12 & $-2.484-2.702$ & $-0.054-0.303$ & $0.326^{\mathrm{ns}}$ & 0.106 \\
\hline \multirow[t]{3}{*}{ PvFL $=(a+b) T L$} & T1(20\%) & 0.6224 & 0.06 & $-0.206-1.451$ & $0.002-0.120$ & $0.456 *$ & 0.208 \\
\hline & T2(25\%) & $\mathbf{0 . 3 5 7 7}$ & 0.08 & $-0.248-0.964$ & 0.035 - 0.119 & $0.675 * *$ & 0.455 \\
\hline & T3(30\%) & -0.0288 & 0.11 & $-1.184-1.127$ & $0.027-0.186$ & $0.552 *$ & 0.305 \\
\hline \multirow[t]{3}{*}{$C F L=(a+b) T L$} & T1(20\%) & 0.5961 & 0.13 & $-1.839-3.031$ & $-0.048-0.298$ & $0.337^{\text {ns }}$ & 0.114 \\
\hline & T2(25\%) & 0.9667 & 0.10 & 0.350 - 1.584 & $0.055-0.140$ & $0.750 * * *$ & 0.563 \\
\hline & T3(30\%) & -3.6927 & 0.43 & $-6.226--1.159$ & $0.255-0.604$ & $0.773 * * *$ & 0.598 \\
\hline \multirow[t]{3}{*}{$\mathbf{A F L}=(a+b) T L$} & T1(20\%) & -0.4030 & 0.15 & $-1.859-1.053$ & $0.046-0.253$ & $0.582 * *$ & 0.339 \\
\hline & T2(25\%) & 0.3706 & 0.08 & $-0.816-1.557$ & $-0.004-0.160$ & $0.427^{\mathrm{ns}}$ & 0.182 \\
\hline & T3(30\%) & -0.6163 & 0.17 & $-2.659-1.427$ & $0.030-0.311$ & $0.515^{*}$ & 0.265 \\
\hline \multirow[t]{3}{*}{$H W=(a+b) T L$} & T1(20\%) & -2.8249 & 0.34 & $-4.664--0.986$ & $0.205-0.467$ & $0.786 * * *$ & 0.618 \\
\hline & T2(25\%) & -0.5268 & 0.22 & $-2.309-1.255$ & $0.100-0.346$ & $0.668 * *$ & 0.446 \\
\hline & T3(30\%) & 0.4164 & 0.15 & $-2.911-3.743$ & $-0.079-0.379$ & $0.309^{\text {ns }}$ & 0.095 \\
\hline \multirow[t]{3}{*}{$E . D=(a+b) T L$} & T1(20\%) & 0.3233 & 0.02 & $-0.211-0.858$ & $-0.020-0.056$ & $0.232 \mathrm{~ns}$ & 0.054 \\
\hline & T2(25\%) & 0.7981 & -0.02 & $0.301-1.296$ & $-0.050-0.018$ & $0.226^{\mathrm{ns}}$ & 0.051 \\
\hline & T3(30\%) & -0.2909 & 0.06 & $-0.627-0.045$ & $0.037-0.083$ & $0.789 * * *$ & 0.623 \\
\hline IntO.D $=(a+b) T L$ & T1(20\%) & 2.1250 & 0.07 & $1.705-2.545$ & $0.037-0.097$ & $0.744 * * *$ & 0.554 \\
\hline & T2(25\%) & 3.5277 & 0.001 & $2.679-4.376$ & $-0.058-0.059$ & $0.004^{\text {ns }}$ & $1.8 \mathrm{E}-05$ \\
\hline & T3(30\%) & 3.0485 & 0.002 & $2.502-3.595$ & $-0.035-0.040$ & $0.029^{n s}$ & 0.001 \\
\hline$P O L=(a+b) T L$ & T1(20\%) & 1.2624 & -0.05 & 0.275 - 2.249 & $-0.123-0.018$ & $0.347 \mathrm{~ns}$ & 0.121 \\
\hline & T2(25\%) & 0.6296 & 0.02 & $0.154-1.106$ & $-0.016-0.049$ & $0.243^{\mathrm{ns}}$ & 0.059 \\
\hline & T3(30\%) & -0.1611 & 0.04 & $-0.662-0.340$ & $0.009-0.078$ & $0.533^{*}$ & 0.284 \\
\hline $\mathrm{DFB}=(\mathrm{a}+\mathbf{b}) \mathrm{TL}$ & T1(20\%) & -0.9154 & 0.13 & $-2.351-0.520$ & $0.033-0.237$ & $0.548 *$ & 0.300 \\
\hline & T2(25\%) & -0.0351 & 0.06 & $-0.583-0.513$ & $0.026-0.102$ & $0.644 * *$ & 0.414 \\
\hline & T3(30\%) & 0.3209 & 0.04 & $-0.729-1.371$ & $-0.030-0.115$ & $0.281^{\mathrm{ns}}$ & 0.079 \\
\hline $2^{\text {nd }} D F B=(a+b) T L$ & T1(20\%) & -0.4364 & 0.05 & $-0.972-0.099$ & $0.009-0.085$ & $0.525 *$ & 0.275 \\
\hline & T2(25\%) & -0.2292 & 0.03 & $-0.620-0.161$ & $0.008-0.062$ & $0.539 *$ & 0.290 \\
\hline & T3(30\%) & 0.4521 & -0.01 & $-0.133-1.037$ & $-0.053-0.028$ & $0.148^{\text {ns }}$ & 0.022 \\
\hline $\mathrm{AFB}=(\mathbf{a}+\mathbf{b}) \mathrm{TL}$ & T1(20\%) & 4.1932 & -0.02 & $1.438-6.948$ & $-0.220-0.172$ & $0.060 \mathrm{~ns}$ & 0.004 \\
\hline & T2(25\%) & 1.2863 & 0.17 & 0.183 - 2.389 & $0.096-0.248$ & $0.746 * * *$ & 0.556 \\
\hline & T3(30\%) & 0.7190 & 0.229 & $-0.950-2.388$ & $0.114-0.344$ & $0.702 * * *$ & 0.493 \\
\hline
\end{tabular}


Table 4: Regression analysis among log of different morphometric parameters of $P$. hypophthalmus toward log total length(cm) at three diet treatments $(n=20)$

\begin{tabular}{|c|c|c|c|c|c|c|c|}
\hline Equations. & $\begin{array}{c}\text { Diet } \\
\text { Variables. }\end{array}$ & $\begin{array}{c}\text { [Relations } \\
\mathbf{a} \\
\end{array}$ & $\begin{array}{c}\text { Irameters. } \\
\text { b }\end{array}$ & $\begin{array}{c}\mathbf{a} \\
(\mathrm{CI.95 \%}) \\
\end{array}$ & $\begin{array}{c}\text { b } \\
(\mathrm{CI} .95 \%) \\
\end{array}$ & r. & $\mathbf{r}^{2}$ \\
\hline \multirow[t]{3}{*}{$\log \cdot W=(a+b) \log T L$} & T1 $(20 \%)$ & -2.0207 & 2.97 & $-3.069--0.973$ & $2.053-3.879$ & $0.849 * * *$ & 0.721 \\
\hline & T2(25\%) & -1.8502 & 2.80 & $-2.607--1.094$ & $2.147-3.451$ & $0.905 * * *$ & 0.819 \\
\hline & T3(30\%) & -0.8996 & 2.00 & $-1.579--0.220$ & $1.418-2.589$ & $0.861 * * *$ & 0.742 \\
\hline \multirow[t]{3}{*}{$\log \cdot K=(a+b) \log T L$} & T1(20\%) & -0.0207 & -0.03 & $-1.069-1.027$ & $-0.947-0.879$ & $\mathbf{0 . 0 1 8}^{\text {ns. }}$ & 0.0003 \\
\hline & T2(25\%) & 0.1498 & -0.20 & $-0.607-0.906$ & $-0.853-0.451$ & $0.151^{\text {ns }}$ & 0.023 \\
\hline & T3(30\%) & 1.1004 & -1.00 & $0.421-1.780$ & $-1.582--0.411$ & $0.644 * *$ & 0.415 \\
\hline \multirow[t]{3}{*}{$\log . F . L=(a+b) \log T L$} & T1(20\%) & 0.1712 & 0.81 & $-0.290-0.633$ & $0.406-1.210$ & $0.705 * * *$ & 0.497 \\
\hline & T2(25\%) & 0.6440 & 0.39 & $0.482-0.806$ & $0.251-0.531$ & $0.811 * * *$ & 0.658 \\
\hline & T3(30\%) & -0.0750 & 1.03 & $-0.845-0.696$ & $0.362-1.689$ & $0.608 * *$ & 0.369 \\
\hline \multirow[t]{3}{*}{$\log . S L=(a+b) \log T L$} & T1(20\%) & 0.2261 & 0.74 & $-0.021-0.473$ & $0.524-0.955$ & $0.862 * * *$ & 0.743 \\
\hline & T2(25\%) & 0.5691 & 0.43 & $0.436-0.702$ & $0.316-0.546$ & $0.880 * * *$ & 0.775 \\
\hline & T3(30\%) & -0.3653 & 1.26 & $-0.759-0.029$ & $0.917-1.596$ & $0.878 * * *$ & 0.771 \\
\hline \multirow[t]{3}{*}{$\log . H L=(a+b) \log T L$} & T1(20\%) & -0.3617 & 0.70 & $-0.888-0.164$ & $0.237-1.154$ & $0.601 * *$ & 0.361 \\
\hline & T2(25\%) & -0.8973 & 1.17 & $-1.637--0.157$ & $0.536-1.812$ & $0.673 * *$ & 0.454 \\
\hline & T3(30\%) & -2.2465 & 2.30 & $-3.107--1.386$ & $1.558-3.040$ & $0.838 * * *$ & 0.702 \\
\hline \multirow[t]{3}{*}{$\log . B D=(a+b) \log T L$} & T1(20\%) & -0.9331 & 1.31 & $-1.468--0.398$ & $0.839-1.771$ & $0.811 * * *$ & 0.658 \\
\hline & T2(25\%) & -0.5987 & 1.08 & $-0.846--0.351$ & $0.869-1.296$ & $0.929 * * *$ & 0.863 \\
\hline & T3(30\%) & 0.0700 & 0.43 & $-0.352-0.492$ & $0.070-0.797$ & $0.508 *$ & 0.259 \\
\hline \multirow[t]{3}{*}{$\log . B G=(a+b) \log T L$} & T1(20\%) & -0.6320 & 1.31 & $-1.167--0.097$ & $0.839-1.771$ & $0.811 * * *$ & 0.658 \\
\hline & T2(25\%) & -0.2977 & 1.08 & $-0.545--0.050$ & $0.869-1.296$ & $0.929 * * *$ & 0.863 \\
\hline & T3(30\%) & 0.3710 & 0.43 & $-0.051-0.793$ & $0.070-0.797$ & $0.508 *$ & 0.259 \\
\hline \multirow[t]{3}{*}{$\log . D F L=(a+b) \log T L$} & T1(20\%) & -2.2923 & 1.99 & $-3.980--0.605$ & $0.516-3.457$ & $0.556 *$ & 0.309 \\
\hline & T2(25\%) & -0.9516 & 1.10 & $-1.821--0.082$ & $0.351-1.850$ & $0.588 * *$ & 0.346 \\
\hline & T3(30\%) & -1.8544 & 1.89 & $-2.550--1.158$ & $1.295-2.493$ & $0.843 * * *$ & 0.710 \\
\hline \multirow[t]{3}{*}{$\log .2^{\text {nd }} D F L=(a+b) \log T L$} & T1(20\%) & -2.2113 & 1.73 & $-3.548--0.875$ & $0.567-2.896$ & $0.593 * *$ & 0.351 \\
\hline & T2(25\%) & -1.3473 & 0.95 & $-2.182--0.513$ & $0.234-1.673$ & $0.549 *$ & 0.301 \\
\hline & T3(30\%) & -0.3003 & 0.04 & $-1.668-1.068$ & $1.134-1.222$ & $0.018^{\mathrm{ns}}$ & 0.0003 \\
\hline \multirow[t]{3}{*}{ Log.Pt.FL $=(a+b) \log T L$} & T1(20\%) & -1.0355 & 1.14 & $-1.509--0.562$ & $0.727-1.552$ & $0.807 * * *$ & 0.652 \\
\hline & T2(25\%) & -0.2860 & 0.49 & $-0.915-0.343$ & $-0.048-1.036$ & $0.411^{\mathrm{ns}}$ & 0.169 \\
\hline & T3(30\%) & -0.8164 & 0.94 & $-2.486-0.854$ & $-0.494-2.382$ & $0.309^{\mathrm{ns}}$ & 0.096 \\
\hline \multirow[t]{3}{*}{ Log.PvFL $=(a+b) \log T L$} & T1(20\%) & -0.4913 & 0.58 & $-1.122-0.139$ & $0.027-1.125$ & $0.461 *$ & 0.213 \\
\hline & T2(25\%) & -0.7063 & 0.75 & $-1.192--0.221$ & $0.336-1.172$ & $0.666 * *$ & 0.443 \\
\hline & T3(30\%) & -0.9202 & 0.95 & $-1.791--0.049$ & $0.197-1.697$ & $0.530 *$ & 0.281 \\
\hline \multirow[t]{3}{*}{$\operatorname{Log.CFL}=(a+b) \log T L$} & T1(20\%) & -0.4638 & 0.73 & $-1.676-0.749$ & $-0.329-1.783$ & $0.323^{\text {ns }}$ & 0.104 \\
\hline & T2(25\%) & -0.3171 & 0.60 & $-0.623--0.011$ & $0.334-0.862$ & $0.746 * * *$ & 0.557 \\
\hline & T3(30\%) & -2.5352 & 2.53 & $-3.673--1.397$ & $1.550-3.509$ & $0.788 * * *$ & 0.620 \\
\hline \multirow[t]{3}{*}{$\log . A F L=(a+b) \log T L$} & T1(20\%) & -1.1411 & 1.19 & $-2.090--0.192$ & $0.367-2.021$ & $0.582 * *$ & $\mathbf{0 . 3 3 8}$ \\
\hline & T2(25\%) & -0.7179 & 0.77 & $-1.665-0.229$ & $-0.047-1.586$ & $0.423^{\mathrm{ns}}$ & 0.179 \\
\hline & T3(30\%) & -1.2707 & 1.32 & $-2.631-0.090$ & $0.15-2.496$ & $0.488^{*}$ & 0.239 \\
\hline \multirow[t]{3}{*}{$\log . H W=(a+b) \log T L$} & T1(20\%) & -2.4876 & 2.41 & $-3.543--1.432$ & $1.488-3.328$ & $0.792 * * *$ & 0.627 \\
\hline & T2(25\%) & -1.0403 & 1.27 & $-1.842--0.238$ & $0.576-1.959$ & $0.672 * *$ & 0.452 \\
\hline & T3(30\%) & -0.5195 & 0.80 & $-2.186-1.147$ & $-0.633-2.237$ & $0.267^{\text {ns }}$ & 0.071 \\
\hline \multirow[t]{3}{*}{$\log -E . D=(a+b) \log T L$} & T1(20\%) & -0.7758 & 0.47 & $-1.879-0.328$ & $-0.493-1.430$ & $0.235^{n s}$ & 0.055 \\
\hline & T2(25\%) & 0.2639 & -0.44 & $-0.806-1.334$ & $-1.365-0.479$ & $0.231^{\text {ns }}$ & 0.053 \\
\hline & T3(30\%) & -2.0865 & 1.59 & $-2.778--1.395$ & $0.997-2.187$ & $0.798 * * *$ & 0.637 \\
\hline Log.IntO.D $=(a+b) \log T L$ & T1(20\%) & 0.1343 & 0.31 & $-0.021-0.290$ & $0.172-0.443$ & $0.747 * * *$ & 0.558 \\
\hline & T2(25\%) & 0.5453 & 0.003 & $0.260-0.831$ & $-0.244-0.249$ & $0.005^{\text {ns }}$ & 2.73E-05 \\
\hline & T3(30\%) & 0.4772 & 0.01 & $0.271-0.683$ & $-0.168-0.187$ & $0.027^{\text {ns }}$ & 0.001 \\
\hline Log.P.OL $=(a+b) \log T L$ & T1(20\%) & 1.3368 & -1.41 & $-0.911-3.585$ & $-3.372-0.546$ & $0.336^{\mathrm{ns}}$ & 0.113 \\
\hline & T2(25\%) & -0.4065 & 0.30 & $-1.067-0.254$ & $-0.272-0.867$ & $0.251^{\mathrm{ns}}$ & 0.063 \\
\hline & T3(30\%) & -1.9592 & 1.41 & $-3.246--0.672$ & $0.299-2.515$ & $0.532 *$ & 0.283 \\
\hline Log.DFB $=(a+b) \log T L$ & T1(20\%) & -2.2923 & 1.99 & $-3.980--0.605$ & $0.516-3.457$ & $0.556^{*}$ & 0.309 \\
\hline & T2(25\%) & -1.2337 & 1.02 & $-1.978--0.489$ & $0.379-1.663$ & $0.619 * *$ & 0.383 \\
\hline & T3(30\%) & -0.7405 & 0.61 & $-1.953-0.472$ & $-0.431-1.657$ & $0.279^{\text {ns }}$ & 0.078 \\
\hline $\log .2^{\text {nd }} D F B=(a+b) \log T L$ & T1(20\%) & -3.6941 & 2.66 & $-6.162--1.227$ & $0.506-4.805$ & $0.522 *$ & 0.272 \\
\hline & T2(25\%) & -3.0257 & 2.12 & $-4.882--1.169$ & $0.520-3.720$ & $0.549^{*}$ & 0.301 \\
\hline & T3(30\%) & 0.2884 & -0.74 & $-2.441-3.018$ & $-3.087-1.614$ & $0.153^{\text {ns }}$ & 0.024 \\
\hline $\log . A F B=(a+b) \log T L$ & T1(20\%) & 0.7055 & -0.10 & $-0.116-1.527$ & $-0.820-0.611$ & $0.072^{\text {ns }}$ & 0.005 \\
\hline & T2(25\%) & -0.1967 & 0.67 & $-0.547-0.154$ & $0.365-0.969$ & $0.738 * * *$ & 0.544 \\
\hline & T3(30\%) & -0.3669 & 0.84 & $-0.853-0.120$ & $0.419-1.257$ & $0.704 * * *$ & 0.495 \\
\hline
\end{tabular}

correlation coefficient $(r), r^{2}$ : coefficient of determination, intercept (a), regression coefficient (b), Cl: confidence intervals, $* * * p<0.001, * * p<0.01, * p<0.05$ 


\section{DISCUSSION}

\section{Growth Study}

The growth study on striped catfish, reared in various crude protein ratios of commercial diets represents that parameters such as average weight gain, average length gain, FCR, live weight gain, daily weight gain condition factor and production; here current study demonstrates considerably significant growth results at investigational fish feed with feed $30 \%$ crude protein followed by feed with $25 \%$ crude protein whereas lowest was documented in fish fed with feed $20 \%$ crude protein. [13] reported that ideal diet protein requirement for lemon fin barb was $30 \%$ that relates recent findings. The protein prerequisite for $P$. hypophthalmus growth is around $32.5 \% \quad[\mathbf{1 4}]$ and 19-30\% [15]. According to [16] FCR of commercial feeds ranges between 1.8 to 22 , which is more than recent findings. According to [17] a low FCR acts as an indicator of improved consumption of feed. [18] reported best production and survival rate on increasing protein ratios (same as above study) on $30 \% \mathrm{CP}$. The average survival rate in recent study varied from $85-98 \%$ which lies close to findings of [19]. According to [20] that protein consumption is relatively insignificant and independent of the dietary protein level. As weight gain by fish is connected to the protein deposition [6]. This justifies the present results, as PER is low with increasing protein levels. Similarly [21] working on $P$. hypophthalmus used commercial feed (29\% $\mathrm{CP})$ according to [22] reports that growth increase through gradual increase in crudeprotien in diet which lies close to recent work $(30 \% \mathrm{CP})$.

\section{Morphometric Analysis}

The work at P.hypophthalmus morphometrics was previously reported by [23]. The phenotypic discerption of various species involves inter-specific or inter-generic, reasons for morphological description to express incidence of growth allometry [24]. The maximum average values for morphometrics in three of the conducted trials were recorded in $30 \% \mathrm{CP}$ (T3). As regression analysis (Table 3) and $\log$ conversion (Table 4) denotes significant positive relationship of TL towards various morphometric characters is in agreement to findings of $[25,26]$. The $\log$ values specify the dissimilar body parts grow contrarily as significance level varies among them. As " $r$ " value of all treatments shows highly significant relationship towards log of mostly morphometric variants as reported by [26]. As the recent findings are near to isometric $(\mathrm{b}=1)$ values detected for $O$. mossambicus by [27] and for T. putitora by [28]. According to 
[26] and [29] owing to deviation in breeds, sexes, physio-chemical conditions, alkalinity, season, biopgeography, and few abiotic factors effects determining the resultant values of ' $\mathrm{b}$ '. The value of $b<3$, obtained in the present study indicates that the growth of $P$. hypophthalmus follows negative allometric trend. This result is consistent with the findings of [30]. Condition factor $(\mathrm{K})$ for Pangasius in the present work (0.83 to 0.88 ) was less than $C$. gibelio (1.021) but more than C.capoeta (0.075) [31]. The lower Kvalues might be due to less feeding intensity. On the other hand, several studies [32-38] reported a non-significant correlation between TL and K-value. In recent findings $\mathrm{K}$ was non-significant $P<0.05$ in $20 \%$ and $25 \%$ CP diet but significant of relation between $\mathrm{TL}$ and $\mathrm{K}$ in $30 \% \mathrm{CP} \operatorname{diet}$ expressing good growth results on recent studies.

\section{CONCLUSION}

It is concluded that the artificial protein diet encourages the protein contents that they manage a generous part in growth and development of fish, to made well-balanced healthy feed. FCR of commercial feeds stood high as expressed in 30\% T3 diet. Small scale farmers of striped-catfish (Pangasianodon hypophthalmus) can have high profit; if they were using commercial- feeds. In addressing this, the certain commercial feeds had the prospective to be utilized as aqua feeds for striped-catfish besides omnivore-fishes with obvious consequences in growth.

\section{REFERENCES}

[1] FAO, Organización de las Naciones Unidas para la Alimentación y la Agricultura. Pesca y Departamento de Acuicultura, 2013.

[2] Oramary S O M, Koramarky D M I, Salih S A, Mustafa A A, Feeding Common Carp Fish (Cyprinus carpio) on Natural Foods (Algae, Phytoplankton, Zooplankton and Others) on Tigris River in Mosul Dam / Duhok, Kurdistan Region of Iraq, J. Aqua Res Devel, 7, 2016, 413.

[3] Phan L P, Bui T M, Nguyen T T, Gooley G J, Ingram B A, Nguyen H V, Phuong T, Nguyen P T, De Silva S S, Current status of farming practices of striped catfish, Pangasianodon hypophthalmus in the Mekong Delta, Vietnam, Aquaculture, 296, 2009, 227-236.

[4] Mzengereza K, Singini W, Msiska O V, Kapute F, Kang'ombe J, Apparent Nutrient Digestibility of Plant Based Diets by Tilapia rendalli (Boulenger, 1896), J.Aqua Res Devel, 7, 2016, 396. 
[5] Deng D, Yong Z, Dominy W, Murashige R, Wilson R P, Optimal dietary protein levels for juvenile Pacific threadfin (Polydactylus sexfilis) fed diets with two levels of lipid, Aquaculture, 316, 2011, 25-30.

[6] Ishtiaq A, Naeem M, Effect of different dietary protein levels on growth performance of Catla catla (Hamilton) reared under polyculture system. Sarhad J. of Agri, 35(3), 2019, 976-984.

[7] Guo Z, Zhu X, Liu J, Han D, Yang Y, Effects of dietary protein level on growth performance, nitrogen and energy budget of juvenile hybrid sturgeon, Acipenser baerii $q \times$ A. gueldenstaedtii §ิ, Aquaculture, 338, 2012, 89-95.

[8] Howe J C, Standard length: not quite so standard, Fisheries Research, 56, 2002, 1-7.

[9] Froese R, Length-weight relationships for 18 less-studied fish species, J. Appl. Ichthyol, 14, 1998, 117-118.

[10] Naeem M, Salam A, Narejo $N$ T, Khokhar M Y, Yar R A, Ishtiaq A, Heavy Metal Detection in Mystus Bleekeri as Bioindicator and Potential Risk of Human Health, Sindh Univ. Res. J.(Sci. Ser.), Vol.44 (2), 2012, 189-194.
[11] Alam M M, Rahman M T, Parween S, Morphometric characters and condition factors of five freshwater fishes from Pagla river of Bangladesh, Int. J. Aqua. Biol, 2(1), 2014, 14-19.

[12] Zamani-Faradonbe M, Eagderi S, Naserabad S S, Length-weight relationships and condition factor of three fish species from Taleghan River (Alborz Province, Iran), J. Adv. Bot. Zool, 2(3), 2015, 1-3.

[13] Suharmili R, Kamarudin M S, Saad C R, Ina-Salwany M Y, Ramezani-Fard E, Mahmud M H, Effects of varying dietary protein level on the growth, feed efficiency and body composition of lemon fin barb hybrid fingerlings. Iran, J. Fish. Sci, 14(2), 2015, 425-435.

[14] Hung $L \mathrm{~T}$, Liem $\mathrm{P} T, \mathrm{Tu} H \mathrm{~T}$, Mariojouls C, Comparing growth and protein requirements for fingerlings of three catfish of the Mekong River (Pangasius bocourti, Pangagasius hypothalmus and Pangasius conchophilus), J. of Aqua in the Tropics, 17(4), 2002, 325-335.

[15] Hung L T, Truc L T T, Huy H P V, Case study on the use of farm-made feeds and commercially formulated pellets for pangasiid catfish culture in the Mekong Delta, Viet Nam: Study and analysis of 
feeds and fertilizers for sustainable aquaculture development. Food and Agriculture Organization of United Nation-FAO, Fisheries Technical, 497, 2007, 363-377.

[16] De Silva S S, Phuong N T, Striped catfish farming in the Mekong Delta, Vietnam: a tumultuous path to a global success, Rev Aquac, 3, 2011, 45-73.

[17] Hillestad M, High-energy diets for Atlantic salmon: effect on growth, feed utilization, product quality and recipient loading. In: Reservoir and culture based fisheries: biology and management. Proc. of an International Workshop held in Bangkok, Thailand from 15-18 February, 2000, 2001, 81pp.

[18] Kpogue D N S, Ayanou G A, Toko I I, Mensah G A, Fiogbe E D, Influence of dietary protein levels on growth, feed utilization and carcass composition of snakehead, Parachanna obscura (Günther, 1861) fingerlings, Afri J. of Zool, Vol.1(4), 2013, 025-030.

[19] Abdel-Zaher A, Mostafa Z M, Ahmad M H, Mousallamy A, Samir A, Effect of using dried Fenugreek seeds as natural feed additives on growth performance, feed utilization, whole-body composition and entropathogenic Aeromonas hydrophila-challinge of monsex Nile tilapia O. niloticus (L) fingerlings, Aust. J. Basic appl. Sci, 3, 2009, 234-1245.

[20] Anguas-Vélez B H, Civera-Cerecedo R, Cadena-Roa M, Guillaume J, MartinezDiaz S F, Studies on the Nutrition of Spotted Sand Bass Paralabrax maculatofasciatus: Effect of the Dietary Protein Level on Growth and Protein utilization in Juveniles Fed Semi purified Diets, J. of World Aqua Soci, $31,2000,580-591$.

[21] Sayeed M A B, Hossain S, Growth performance of thai pangus (pangasius hypophthalmus) in polyculture system using different supplementary feeds, Univ. j. zool. Rajshahi Univ, Vol. 27, 2008, 59-62.

[22] Kader M A, Hossain M A, Hossain M D A, comparative study on the effect of commercial fish feeds on the growth of Thai pangas, Pangasius hypophthalmus, Bangla. Fish. Res, 7(1), 2003, 53-58.

[23] Tosin V O, Ivan C C K, Anuar H, Thumronk A, Sherif M S, Morphological characterization of the progenies of pure and reciprocal crosses of Pangasianodon hypophthalmus (Sauvage, 1878) and Clarias gariepinus (Burchell,1822), SCI. REPO, 8, 2018, 3827. 
[24] Costa C, Shape studies in Ichthyology: geometric morphometric tools and outlines fitting methods (Lo studio della forma in ittiologia: morfometrid geometrica ed analisi dei profili), $\mathrm{PhD}$. Thesis discussed at the University of Rome "Tor Vergata", 2004, 177pp.

[25] Naeem M, Narejo N T, Salam A, Rasool S A, Khalid M, Ishtiaq A, LengthWeight Relationships of Juvenile Pangasius with Special Reference to Body Size and Condition Factor, Sindh Univ. Res. Jour, (Sci. Ser.) Vol. 47(3), 2015, 523-526.

[26] Khalid M, Naeem M, Morphometric relationship of length-weight and lengthlength of farmed Ctenopharyngodon idella from Muzaffar Garh, Southern Punjab, Pakistan, Punjab Univ. J. Zool, 32(1), 2017, 57-64.

[27] Naeem M, Salam A, Baby R, Ali M, Ishtiaq A, Ashraf $\mathrm{M}$, Length-Weight relationship of female population of farmed Oreochromis mossambicus in relation to body size and condition factor from Pakistan. International Conference on Bioscience, Biochemistry and Bioinformatics (ICBBB 2011), 26-28 February2011, Singapore, 2011a, 360363.
[28] Naeem M, Salam A, Ashraf M, Khalid M, Ishtiaq A, External morphometric study of hatchery reared mahseer (Tor putitora) in relation to body size and condition factor, Afr. J. Biotechnol, 10(36), 2011b, 7071-7077.

[29] Chu W S, Hou Y Y, Ueng Y, Wang J P, Length-weight relationships of fishes in Chi-gu black-spoonbill reserve area of Taiwan, J. Appl. Ichthyol, 28, 2012, 150-151.

[30] Ren B, Ma Y. W, Turson, Guo Y, Zhang R M, A Bu-du, Au Z Z, Liu J, The study on the biology of Triplophysa (Hedinichthgs) yarkandensis (Day) in Akesu River, Chinese J. Fish, 17, 2004, 46-52 (Chinese Jounral with English abstract).

[31] Radkhah A, Eagderi S, Length-weight and length-length relationships and condition factor of six cyprinid fish species from Zarrineh River (Urmia Lake basin, Iran), Iran. J. Ichthyol, 2(1), 2015, 61-64.

[32] Nazir MI, Idrees I, Danish P, Ahmad S, Ali Q, Malik A. Potential of water hyacinth (Eichhornia crassipes L.) for phytoremediation of heavy metals from waste water . Biol. Clin. Sci. Res. $J, 2020,2020$ : e006. 
[33] Khalil, R., Ali, Q., Hafeez, M.M., Malik, A. Phenolic acid profiling by RP-HPLC: evaluation of antibacterial and anticancer activities of Conocarpus erectus plant extracts. Biol. Clin. Sci. Res. J., 2020, 2020: e010.

[34] Naeem M, Ishtiaq A, Proximate composition of Mystus bleekeri in relation to body size and condition factor from Nala Dail, Sialkot, Pakistan, Afri. J. of Biotech, Vol. 10(52), 2011, 10765-10763.

[35] Hayat S, Ramzan M, Zafarullah M, Ahmad I, Ali Q, Malik A. Conservation of an endangered carnivorous fish rita rita through induced breeding. Biol. Clin. Sci. Res. J, 2020, 2020: e004.
[36] Hayat, S., Naeem, M,. Ramzan, M., Zafarullah, M., Ahmad, I., Ali, Q., Malik, A. Study on zooplankton diversity during spring season in Chashma Lake, Pakistan. Biol. Clin. Sci. Res. J., 2020, 2020: e009.

[37] Ahmad, S., and Hera, Z. (2020). Effects of carbosulfan on the biology of bird cherry oat aphid. Biol. Clin. Sci. Res. J., 2020, 2020: e015.

[38] Yaqoob S, Fatima N, Khan S, Ali Q, Hafeez MM, Malik A., Begomoviruses and betasatellites associated with CLCuD. Biol. Clin. Sci. Res. J, 2020, 2020: e002. 